
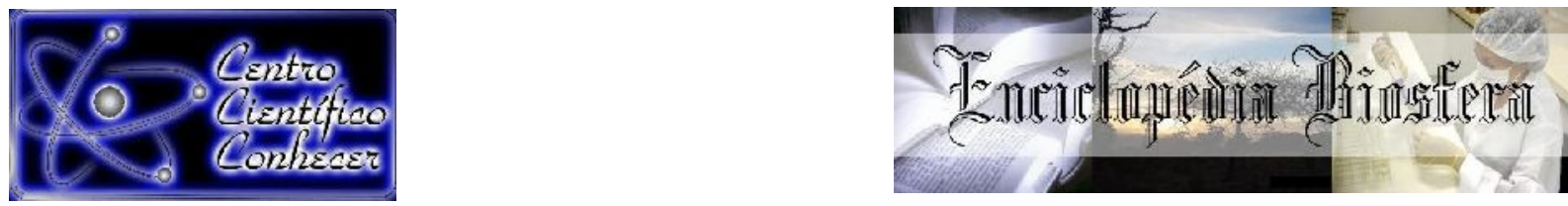

\title{
EFEITO DA CONCENTRAÇÃO DO SOLVENTE NA CAPACIDADE DE EXTRAÇÃO DE PIGMENTOS ANTOCIANINAS
}

Mayara Marques Lima ${ }^{1^{*}}$, Wallinson Pires da Cruz ${ }^{2}$, Beatriz Soares Ranke ${ }^{1}$, Ricardo Pereira Moraes ${ }^{3}$, Rosemary Maria Pimentel Coutinho ${ }^{4}$

1 Bacharel em Engenharia Ambiental, Universidade do Estado do Pará, Marabá-PA, Brasil. *Email: maymarques1304@gmail.com

2 Licenciado em Química, Universidade do Estado do Pará, Marabá-PA, Brasil.

3 Graduando em Agronomia, Universidade Federal do Sul e Sudeste do Pará, Marabá-PA, Brasil.

4 Professora Doutora do Instituto Federal de Educação, Ciência e Tecnologia do Pará Campus Marabá Industrial, Marabá-PA, Brasil.

Recebido em: 15/11/2020 - Aprovado em: 15/12/2020 - Publicado em: 30/12/2020 DOI: 10.18677/EnciBio_2020D15

\begin{abstract}
RESUMO
As flores das espécies Allamanda blanchetii A. DC., Bougainvillea glabra Choisy var. alban. var., Catharanthus roseus (L.) G. Don e Macroptilium lathyroides (L.) Urb., são vegetais que possuem poucos estudos sobre seus metabolitos secundários. As antocianinas são metabolitos secundários pertencentes a classe dos flavonoides e são conhecidas por suas propriedades antioxidantes. O estudo objetivou avaliar diferentes concentrações de etanol para extração de antocianinas totais e avaliar duas técnicas de quantificação. Os extratos foram produzidos nas concentrações de etanol a 70\%, 85\% e $95 \%$ respectivamente, acidificados com ácido clorídrico 1,5N na proporção 85:15. Para determinação dos teores de antocianinas foi utilizado espectrofotômetro Uv-Vis, para obtenção das absorbâncias no comprimento de onda a $535 \mathrm{~nm}$. Os melhores resultados foram observados na concentração etanólica a 95\% em ambos os métodos (pH único e $\mathrm{pH}$ diferencial), sendo que o extrato fitoquimicos da espécie $M$. lathyroides apresentou maior conteúdo de antocianinas $\left(1142,40 \mathrm{mg} 100 \mathrm{~g}^{-1}\right.$ e $\left.1254,09 \mathrm{mg} .100 \mathrm{~g} \mathrm{~g}^{-1}\right)$, seguido por A. blanchetii (302,94 mg.100 g e 250,13 mg.100 g $\left.{ }^{-1}\right)$, C. roseus $\left(139,84 \mathrm{mg}^{-1} 100 \mathrm{~g} \mathrm{~g}^{-1}\right.$ e $203,93 \mathrm{mg} .100 \mathrm{~g}^{-1}$ ) e B. glabra $\left(127,62 \mathrm{mg} .100 \mathrm{~g}^{-1}\right.$ e $\left.85,67 \mathrm{mg} .100 \mathrm{~g}^{-1}\right)$. Os resultados deste estudo poderão colaborar com o desenvolvimento de novos produtos para a indústria de alimentos, farmacêutica ou cosmética, com satisfatória atividade bioativa a base destes vegetais.
\end{abstract}

PALAVRAS-CHAVE: flores; extrato fitoquímico; metabolitos secundários.

\section{EFFECT OF SOLVENT CONCENTRATION ON THE EXTRACTION CAPACITY OF ANTHOCYANIN PIGMENTS}

\begin{abstract}
The flowers of the species Allamanda blanchetii A. DC., Bougainvillea glabra Choisy var. Alban. var., Catharanthus roseus (L.) G. Don and Macroptilium lathyroides (L.) Urb., are vegetables that have few studies on their secondary metabolites. Anthocyanins are secondary metabolites belonging to the flavonoid class and are known for their
\end{abstract}


antioxidant properties. The study aimed to evaluate different concentrations of ethanol for the extraction of total anthocyanins and to evaluate two quantification techniques. The extracts were produced in the concentrations of $70 \%, 85 \%$ and $95 \%$ ethanol, respectively, acidified with $1.5 \mathrm{~N}$ hydrochloric acid in the ratio $85: 15$. To determine the anthocyanin levels, a Uv-Vis spectrophotometer was used to obtain the absorbances at wavelength at $535 \mathrm{~nm}$. The best results were observed in the $95 \%$ ethanolic concentration in both methods (single $\mathrm{pH}$ and differential $\mathrm{pH}$ ), with the phytochemical extract of the species $M$. lathyroides having a higher anthocyanin content $(1142.40$ mg.100 g-1 and 1254 , $09 \mathrm{mg} .100 \mathrm{~g}-1$ ), followed by A. blanchetii (302.94 mg.100 g-1 and $250.13 \mathrm{mg} .100 \mathrm{~g}-1$ ), C. roseus (139.84 mg.100 g-1 and $203.93 \mathrm{mg} .100 \mathrm{~g}-1$ ) and B. glabra (127.62 mg.100 g-1 and $85.67 \mathrm{mg} .100 \mathrm{~g}-1$ ). The results of this study may collaborate with the development of new products for the food, pharmaceutical or cosmetic industry, with satisfactory bioactive activity based on these vegetables.

KEYWORDS: flowers; phytochemical extract; secondary metabolites.

\section{INTRODUÇÃO}

Pigmentos naturais são encontrados em diversas flores e frutos de várias plantas e, vem sendo alvo de muitos estudos, não somente por conferir cor, mas por suas propriedades bioativas benéficas à saúde. Estudos mostram que a redução de doenças crônicas está relacionada à ingestão de alimentos ricos em antioxidantes. Dentre os principais antioxidantes presentes nos vegetais estão as vitaminas $C$ e $E$, os carotenoides e os compostos fenólicos, em especial os flavonoides (MODESTO JUNIOR et al., 2016; MENESES-MARENTES et al., 2019).

Os flavonoides são considerados potentes antioxidantes naturais, isso se deve aos grupos hidroxila ligados à estrutura do anel aromático. A capacidade antioxidante dos flavonoides geralmente aumenta com o acréscimo dos grupos hidroxila resultados do favorecimento na deslocalização de elétrons nos núcleos aromáticos, permitindo assim a estabilidade do radical, que impedem a oxidação de alimentos, em especial os lipídeos (MENEZES et al., 2015; BURGOS et al., 2016).

As antocianinas são metabólitos secundários pertencentes a classe dos flavonoides e vem sendo utilizada na indústria como corante natural. Por pertencer a este grupo, a atividade antioxidante das antocianinas é entendida como a capacidade de inibir processos de degradação oxidativa, devido a sua composição química, assim como também sua atividade biológica que trazem diversos benefícios a saúde (HURTADO; CHARFUELAN, 2019).

Vários métodos e solventes vêm sendo usado para a extração de antocianinas, o uso do solvente correto possibilita a obtenção de maiores teores destes compostos, sendo os principais extratores a água, o etanol e a acetona, estes solventes são considerados os mais seguros para produção de alimentos e medicamentos, pois possuem baixa toxicidade para o uso humano e animal, assim como para o meio ambiente (MENEZES FILHO et al., 2019).

As flores das espécies Allamanda blanchetii A. DC., Bougainvillea glabra Choisy var. alban. var., Catharanthus roseus (L.) G. Don e Macroptilium lathyroides (L.) Urb., são vegetais que possuem escassos estudos sobre suas propriedades químicas, sendo válida a investigação de metabolitos secundários existentes nessas espécies (ARAÚJO et al., 2011; ALTHAUS-OTTMANN et al., 2011; GONÇALVES; PASA, 2015; LIMA et al., 2020). 
O objetivo deste trabalho foi avaliar o conteúdo total de antocianinas em diferentes concentrações de etanol e avaliar duas técnicas de quantificação desses compostos, com o intuito de contribuir com a disseminação de informação acerca de novas fontes de antocianinas.

\section{MATERIAL E MÉTODOS}

Este trabalho foi realizado no Laboratório de Química do Instituto Federal do Pará (IFPA) - Campus Marabá Industrial, na cidade de Marabá-PA, Brasil. A coleta das flores $A$. blanchetti e $B$. glabra foi realizada no viveiro da Secretaria de Meio Ambiente de Marabá (SEMMA) e as flores de $M$. lathyroides e $C$. roseus foram coletadas do jardim de uma residência das proximidades do IFPA, as mesmas foram coletadas aleatoriamente, no turno da manhã em novembro de 2018.

Todas as flores passaram por uma triagem manual, para a separação do material de interesse (pétalas), logo após foram lavadas com água destilada, secadas ao ar livre, pesadas e imersas em solução extratora.

\section{Extrato Concentrado}

Os extratos foram feitos conforme metodologia adaptada de Fuleki e Francis (1968a; 1968b). As amostras (pétalas de flores) foram trituradas e pesadas $(2,0 \mathrm{~g})$, as mesmas foram transferidas para um vidro âmbar e adicionou-se $50 \mathrm{~mL}$ de solvente extrator etanol $70 \%-\mathrm{HCl} 1,5 \mathrm{~N}(85: 15)$. O material permaneceu em repouso por 24 horas a $5^{\circ} \mathrm{C}$, ao abrigo da luz, para extração. Após esse período foi realizada filtração simples, e logo após, o extrato foi transferido para balão volumétrico de $100 \mathrm{~mL}\left(\mathrm{~V}_{\mathrm{EC}}\right)$, envolto em papel alumínio, tendo seu volume completado com o solvente extrator, formando o Extrato Concentrado (EC). Para as concentrações de $85 \%$ e 95\% de etanol, ocorreu da mesma maneira, diferenciando apenas as concentrações de etanol (Figura $1)$.

FIGURA 1. Fluxograma do processo de extração do pigmento.

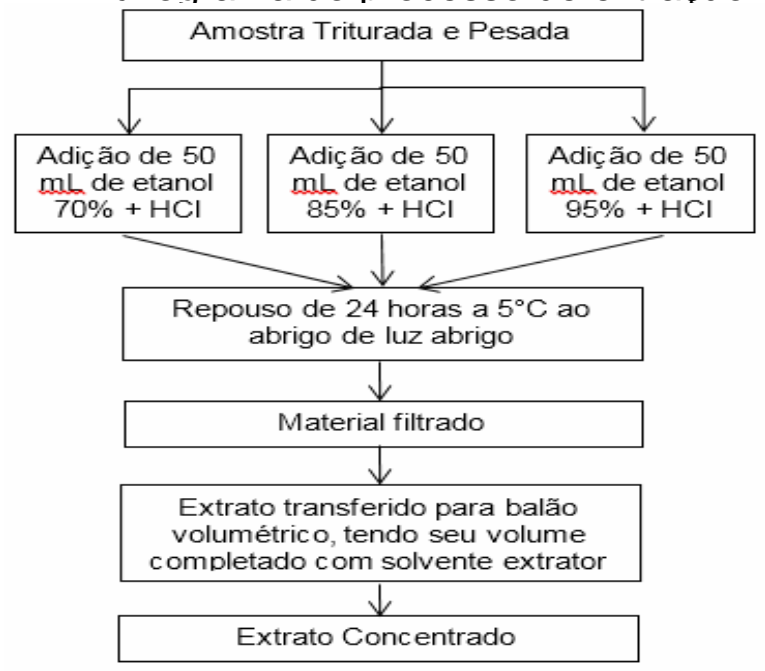

Fonte: Autores (2018). 


\section{Quantificação de Antocianinas Totais}

A quantificação das antocianinas totais foi realizada através dos métodos de $\mathrm{pH}$ único e pH diferencial adaptados a partir dos trabalhos realizados por Fuleki e Francis (1968a; 1968b) e Teixeira et al., (2008). As Absorbâncias foram obtidas em espectrofotômetro Uv-Vis da marca HACH, modelo DR3900 no comprimento de onda a $535 \mathrm{~nm}$. A quantidade de antocianinas totais foi expressa em $\mathrm{mg} / 100 \mathrm{~g}$ e as análises foram realizadas em triplicata.

Para a realização do método de $\mathrm{pH}$ único (Figura 2), transferiu-se uma alíquota $\left(V_{\text {alq }}\right)$ do extrato concentrado para um balão volumétrico $\left(\mathrm{V}_{\mathrm{Ed}}\right)$, e o volume foi completado com os respectivos solventes extratores, formando o extrato diluído. Antes da realização da leitura das absorbâncias, os valores dos brancos (Soluções de etanol $+\mathrm{HCl} 1,5 \mathrm{~N}$ ) foram zerados.

O método de $\mathrm{pH}$ Diferencial, (Figura 2), consistiu na transferência de uma alíquota do extrato concentrado para dois balões volumétricos $\left(V_{E d}\right)$. Um dos balões volumétricos foi completado com a solução tampão de $\mathrm{KCl}(0,2 \mathrm{~N})$ e $\mathrm{HCl}(0,2 \mathrm{~N})$ na proporção de 25:67 para o pH 1 e o outro balão foi completado com uma solução de Acetato de Sódio $\left(\mathrm{C}_{2} \mathrm{H}_{3} \mathrm{NaO}_{2}(1 \mathrm{~N})\right)$, $\mathrm{HCl}$ e água na proporção 100:60:90. Antes das leituras das absorbâncias, os valores dos brancos (soluções de pH 1 e pH 4,5) foram zerados.

FIGURA 2. Fluxograma do preparo do Extrato Diluído.

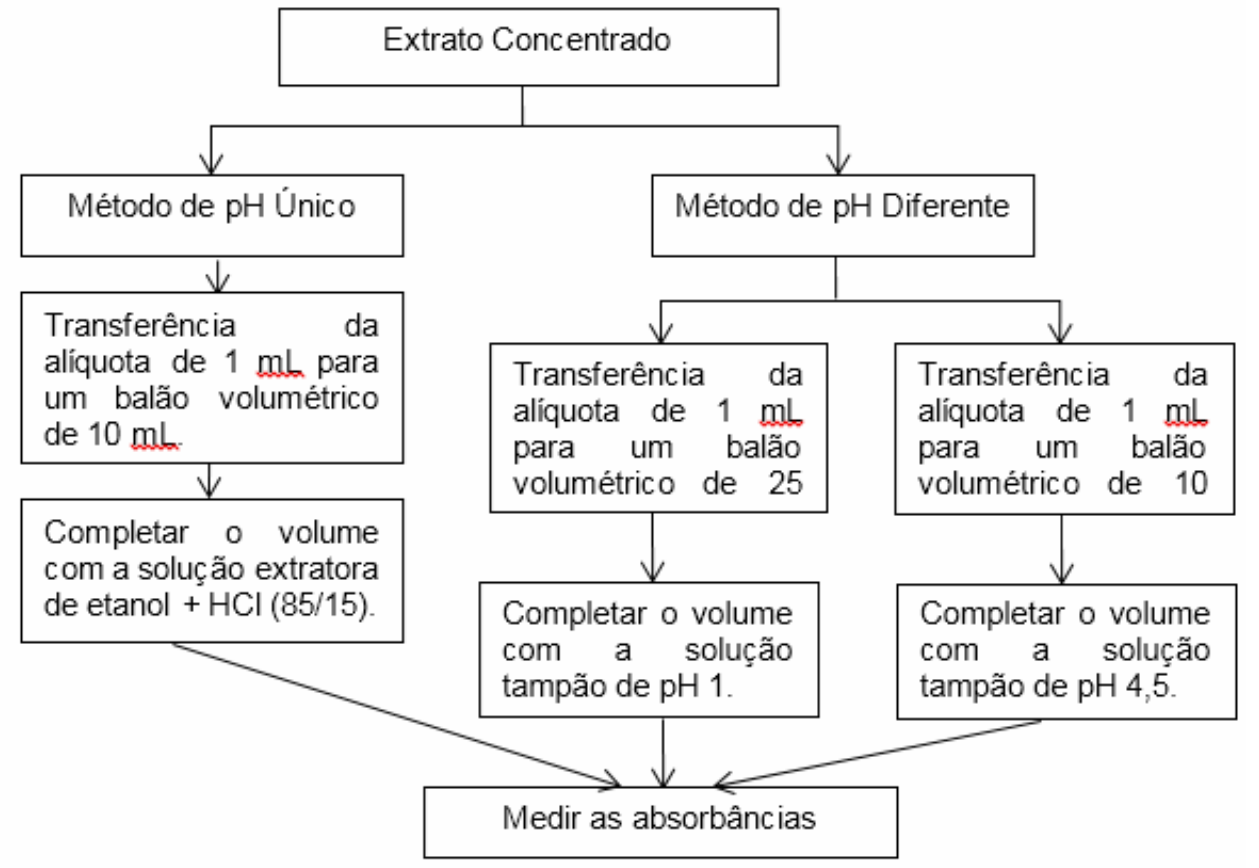

Fonte: Autores (2018).

O teor de Antocianinas Totais (AntT) foi obtido conforme Fórmula 01, adotou-se o coeficiente de Extinção médio de valor de 982 para o método de $\mathrm{pH}$ único e para o método de $\mathrm{pH}$ diferencial 873 e 775 para o $\mathrm{pH} 1$ e pH 4,5, respectivamente. 


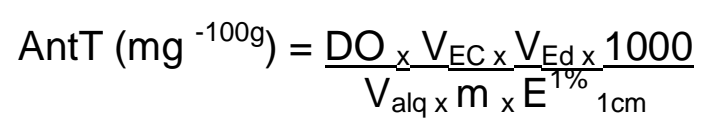

Onde,

DO: Densidade ótica do extrato diluído, para o pH Único usa-se a medição direta obtida no espectrofotômetro, quanto ao pH diferencial realiza-se a diferença entre as densidades óticas obtidas para o $\mathrm{pH} 1$ e para o $\mathrm{pH} 4,5$.

$\mathrm{V}_{\mathrm{EC}}$ : Volume do extrato concentrado

$V_{E d}$ : Volume do extrato diluído

$V_{\text {alq }}$ : Volume da alíquota utilizada na diluição do extrato concentrado

m: Massa da amostra

$\mathrm{E}^{1 \%}{ }_{1 \mathrm{~cm}}$ : Coeficiente de Extinção Médio de antocianinas

\section{Delineamento Experimental}

O experimento foi conduzido em Delineamento Inteiramente Casualizado, em arranjo fatorial $4 \times 3 \times 2$ (quatro espécies de flores, três concentrações de etanol e dois métodos de análise). A análise dos dados da quantificação de antocianinas foi realizada pela aplicação da ANOVA e o teste de Tukey, visando identificar diferenças significativas entre as médias. O nível de significância considerado para a diferença entre as médias foi de $5 \%(p<0,05)$. Todas as análises foram realizadas em triplicata e os resultados apresentados como média \pm desvio padrão, assim como tabelas foram elaboradas em software Microsoft Excel 2016.

\section{Quantificação de Antocianinas}

\section{RESULTADOS E DISCUSSÃO}

Com os dados obtidos neste estudo, verificou-se que a solução etanólica a 95\% $\mathrm{HCl} 1,5 \mathrm{~N}$, obteve a melhor extração para todas as fontes de antocianinas em ambos os métodos de quantificação (Tabela 1).

TABELA 1. Antocianinas totais de extratos de flores*

\begin{tabular}{|c|c|c|c|c|}
\hline \multirow{2}{*}{ Espécies } & \multirow{2}{*}{$\begin{array}{c}\text { Concentração de } \\
\text { Etanol }\end{array}$} & \multicolumn{2}{|c|}{ Antocianinas Totais $\left(\mathrm{mg} .100 \mathrm{~g}^{-1}\right)$} & \multirow[b]{2}{*}{ Valor de $\mathbf{P}$} \\
\hline & & pH Único & pH Diferencial & \\
\hline \multirow{3}{*}{ A. blanchetti } & $70 \%$ & $144,94 \pm 1,06^{A}$ & $94,35 \pm 8,30^{A}$ & 0,0004 \\
\hline & $85 \%$ & $176,22 \pm 5,58^{A}$ & $157,96 \pm 2,02^{B}$ & 0,0060 \\
\hline & $95 \%$ & $302,94 \pm 31,42^{B}$ & $250,13 \pm 8,34^{c}$ & 0,0481 \\
\hline \multirow{3}{*}{ B. glabra } & $70 \%$ & $34,11 \pm 0,51^{\mathrm{c}}$ & $36,9 \pm 0,90^{\mathrm{D}}$ & 0,0095 \\
\hline & $85 \%$ & $100,98 \pm 1,27^{\mathrm{D}}$ & $67,08 \pm 1,06^{\mathrm{AD}}$ & $<0,0001$ \\
\hline & $95 \%$ & $127,62 \pm 1,28^{\mathrm{AD}}$ & $85,67 \pm 8,28^{A}$ & 0,0009 \\
\hline \multirow{3}{*}{ C. roseus } & $70 \%$ & $100,47 \pm 0,77^{\mathrm{D}}$ & $78,74 \pm 2,76^{\mathrm{A}}$ & 0,0002 \\
\hline & $85 \%$ & $116,08 \pm 1,02^{A D}$ & $138,28 \pm 2,54^{B}$ & 0,0001 \\
\hline & $95 \%$ & $139,84 \pm 17,51^{\mathrm{A}}$ & $203,93 \pm 1,48^{\mathrm{E}}$ & 0,0032 \\
\hline \multirow{3}{*}{ M. lathyroides } & $70 \%$ & $207,73 \pm 1,52^{\mathrm{E}}$ & $215,30 \pm 2,51^{\mathrm{E}}$ & 0,0111 \\
\hline & $85 \%$ & $437,03 \pm 14,02^{\mathrm{F}}$ & $568,16 \pm 14,74^{F}$ & 0,0003 \\
\hline & $95 \%$ & $1142,40 \pm 21,56^{\mathrm{G}}$ & $1254,09 \pm 1,45^{\mathrm{G}}$ & 0,0008 \\
\hline
\end{tabular}

${ }^{\star}$ Valores expressos como média \pm desvio padrão, letras diferentes na mesma coluna apesentam diferença significante entre si (Teste de Tukey, a 5\% de significância). 
Apesar de não haver diferença significativa entre os métodos avaliados ( $p<$ $0,05)$, as espécies $A$. blanchetii e $B$. glabra obtiveram valores superiores no método de $\mathrm{pH}$ único, já as espécies $C$. roseus e $M$. lathyroides obtiveram valores superiores no método de $\mathrm{pH}$ diferencial.

Observou-se uma ordem crescente no teor de antocianinas entre as concentrações de etanol tanto no método de $\mathrm{pH}$ único quanto no método de $\mathrm{pH}$ diferencial, sendo a espécie $M$. lathyroides $(1142,40 \pm 21,56$ e 1254,09 $\pm 1,45)$ a que apresentou maior teor de antocianinas, seguida por $A$. blanchetti $(302,94 \pm 31,42$ e $250,13 \pm 8,34 \mathrm{C})$, C. roseus $(139,84 \pm 17,51$ e $203,93 \pm 1,48)$ e $B$. glabra $(127,62 \pm 1,28$ e 85,67 $\pm 8,28)$ que apresentaram menores teores entre as espécies estudadas.

É importante ressaltar que a maior eficiência na concentração de etanol a 95\%, pode ser justificada pelo uso do etanol em meio acidificado que possibilita maior extração do pigmento antocianina, onde há maior interação entre as moléculas de antocianinas e o etanol (BURGOS et al., 2016).

Em estudo realizado por Rockenbach et al. (2008), quando avaliaram a influência do solvente no conteúdo de antocianinas na Ancelota e Tannat, observaram que houve um aumento progressivo do pigmento entre as concentrações de etanol a $30 \%, 50 \%$ e $70 \%$, exceto na concentração de etanol $100 \%$ e na água que tiveram uso isolado, a quantidade de antocianinas reduziu significativamente.

Para Alcântara et al. (2019), ao utilizarem um solvente isoladamente a quantidade de compostos bioativos pode ser menor do que ao uso combinado de dois ou mais solventes durante a extração. Estes autores frisam ainda, que o uso misto de solventes depende da finalidade para a qual o analista deseja extrair, dependendo do objetivo pode-se extrair quantidades superiores dos compostos. No caso deste estudo foram consideradas satisfatórias as quantidades extraídas, já que o etanol possui baixa toxicidade, sendo um dos mais indicados para uso na indústria.

Teores de antocianinas similares às encontradas neste estudo foram obtidas em recentes trabalhos publicados por Lima et al. (2019; 2020), que relataram extrair das pétalas das mesmas espécies deste estudo, usando solvente etanol 95\% acidificado, com valores médios de antocianinas iguais a $221,69 \pm 0,36$ na $A$. blanchetti, $71,70 \pm$ 0,59 na $B$. glabra, $120,50 \pm 2,64$ na $C$. roseus e $1129,90 \pm 0,59$ na $M$. lathyroides.

A quantidade de antocianinas nas flores avaliadas nesta pesquisa, foram superiores aos valores encontrados por Silva et al., (2016) nas flores de hibisco

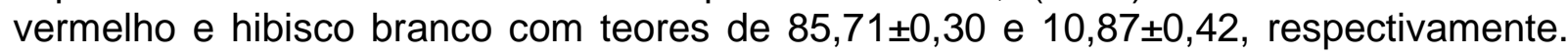
As flores de hibisco já são utilizadas na culinária e são relatadas como plantas medicinais em livros, isso denota que as flores pesquisadas devem ter estudos aprofundados.

Em trabalho realizado por Costa et al., (2014), obtiveram teores de antocianinas significantes na rosa vermelha, nas flores de maracujá e nas flores de capuchicha vermelha, com teores médios de 405,7 mg.100 g $\mathrm{g}^{-1}$; 378,5 mg.100 $\mathrm{g}^{-1} \mathrm{e}$ aproximadamente $270 \mathrm{mg} .100 \mathrm{~g}^{-1}$, respectivamente.

\section{CONCLUSÃO}

Os extratos obtidos a partir de espécies de flores apresentaram valores satisfatórios de antocianinas totais em ambos os métodos utilizados, sendo a concentração de etanol a $95 \%$ a mais eficiente para todas as espécies. A fonte com maior quantidade de antocianinas foi a $M$. lathyroides com 1254,09 mg.100 g ${ }^{-1}$. Vale 
ressaltar que a busca por novas fontes de compostos bioativos é de suma importância para a elaboração de novos produtos naturais e renováveis.

Pode-se concluir neste estudo, que a escolha de um solvente etanólico na concentração ideal e acidificado, proporciona uma maior extração dos pigmentos antocianinas, este resultado garante que um produto desenvolvido a partir das fontes estudadas seja aplicado em diversos processos industriais de indústrias como as de alimentos, farmacêuticas e cosméticas.

\section{REFERÊNCIAS}

ALCÂNTARA, M. A.; POLARI, I. L. B.; MEIRELES, B. R. L. A.; LIMA, A. E. A.; JUNIOR, J. C. S. et al. Effect of the solvent composition on the profile of phenolic compounds extracted from chia seeds. Food Chemistry, v. 275, p. 489-496, 2019. DOI: https://doi.org/10.1016/j.foodchem.2018.09.133

ALTHAUS-OTTMANN, M.M.; CRUZ, M.J.R.; FONTE, N.N. Diversidade e uso das plantas cultivadas nos quintais do Bairro Fanny, Curitiba, PR, Brasil. Revista Brasileira Biociências, v 9, n. 1, p.39-49, 2011. Disponível em: <http://www.ufrgs.br/seerbio/ojs/index.php/rbb/article/view/1646>

ARAÚJO, L.D.A.; QUIRINO, Z.G.M.; MACHADO, I.C. Fenologia reprodutiva, biologia foral e polinização de Allamanda blanchetii, uma Apocynaceae endêmica da Caatinga. Revista Brasileira de Botânica, v 34, n. 2, p. 211-222, 2011. DOI: 10.1590/S010084042011000200008

BURGOS, K. S. C.; REYES, S. G. R; YUPANQUI, M. L. G. Antocianinas totales y capacidad antioxidante in vitro de extractos de diferente grado etanólico del fruto de Vacciniumcorymbosum "Arándano". UCV - Scientia, v. 8, n. 1, p. 44-48, 2016. Disponível em: <http://181.224.246.204/index.php/UCV-SCIENTIA/article/view/1008>

COSTA, L. C; RIBEIRO, W. S.; BARBOSA, J. A. Compostos Bioativos e Alegações de Potencial Antioxidante de Flores de Maracujá, Cravo Amarelo, Rosa e Capuchinha. Revista Brasileira de Produtos Agroindustriais, Campina Grande, v.16, n.3, p.279289, 2014. DOI: 10.15871/1517-8595/rbpa.v16n3p279-289

FULEKI, T.; FRANCIS, F. J. Quantitative methods for anthocyanins. 2. Determination of total anthocyanin and degradation Index for cranberry juice, Journal of Food Science, v. 33, n. 1, p. 78-83, 1968b. DOI: http://dx.doi.org/10.1111/j.1365-2621.1968.tb00888.x

FULEKI, T.; FRANCIS, F. J. Quantitative methods for anthocyanins. 1. Extraction and determination of total anthocyanin in Cranberries. Journal of Food Science, v.33, n.1, p.72-77, 1968a. DOI: https://doi.org/10.1111/j.1365-2621.1968.tb00887.x

GONÇALVES, K.G.; PASA, M.C. A etnobotânica e as plantas medicinais na Comunidade Sucuri, Cuiabá, MT, Brasil. Interações, v. 16, n. 2, p. 245-256, 2015. DOI: http://dx.doi.org/10.1590/1518-70122015201

HURTADO, N. H.; CHARFUELAN, C. Contribución a la Caracterización y Evaluación de la Actividad Antioxidante de las Antocianinas del Fruto de Ivilan (Monnina Obtusifolia H.B.K). Información Tecnológica, v. 30, n. 5, p. 81-90, 2019. DOI: 
http://dx.doi.org/10.4067/S0718-07642019000500081

LIMA, M. M; ALMEIDA, J. N. S.; CRUZ, W. P.; MORAES, R. P., COLLINGE, M. D. R.; COUTINHO, R. M. P. Estudo da espécie Macroptillium Lathyroides como uma espécie com propriedade bioativa, uma flor comestível. In: Silva, F. F. Prática e pesquisa em ciência e tecnologia de alimentos 2. 2. Ed. Ponta Grossa, PR: Atena, 2020. p. 1-9. DOI 10.22533/at.ed.2702006039

LIMA, M. M; ALMEIDA, J. N. S.; CRUZ, W. P.; SILVA, M. N.; COUTINHO, R. M. P. Quantificação de Antocianinas Totais Presentes nas Flores de Espécies Vegetais. In: Silva-Matos, R. R. S.; Souza, G. M. M; Costa, A. C. S. Meio Ambiente Inovação com Sustentabilidade2. 2. Ed. Ponta Grossa, PR: Atena, 2019. p. 89-98. DOI 10.22533/at.ed.4611901101

MENESES-MARENTES, N. A; HERRERA-RAMÍREZ, E. J; TARAZONA-DÍAZ, M. P. Caracterización y estabilidad de un extracto rico en antocianinas a partir de corteza de gulupa. Revista Colombiana Quimica, v. 48, n. 2, p. 27-32, 2019. DOI: http://dx.doi.org/10.15446/rev.colomb.quim.v48n2.76682

MENEZES, M. A. G.; OLIVEIRA NETO, F. B.; BERTINI, L. M.; ALVES, L. A.; SILVA, F. F. M. Quantificação de antocianinas dos extratos de embiratanha (Pseudobombax marginatum). HOLOS, v. 1, n. 31, p. 30-35, 2015. DOI: 10.15628/holos.2015.2459

MENEZES FILHO, A. C. P.; OLIVEIRA FILHO, J. G.; SOUZA, J. C. P.; CASTRO, C. F. S. Avaliação de diferentes solventes para extração dos compostos fenólicos totais da farinha do fruto Calabura (Muntingia calabura Linn.). Biota Amazônia, v. 9, n. 2, p. 2123, 2019. DOI: http://dx.doi.org/10.18561/2179-5746/biotaamazonia.v9n2p21-23

MODESTO JUNIOR, E. N.; SOARES, S. S.; GOMES, P. W. P.; RIBEIRO, C. F. A.; DA SILVA, R. M. V. Estudo do armazenamento da polpa do fruto ginja Eugênia uniflora $L$. e sua influencia nos teores de ácido ascórbico e antocianinas. Scientia Plena, v. 12, n. 6 , p. 1-8, 2016. Doi: 10.14808/sci.plena.2016.069932

ROCKENBACH, I. I; SILVA, G. L. RODRIGUES, E.; KUSKOSKI, E. M.; FEIT, R. Influência do solvente no conteúdo total de polifenóis, antocianinas e atividade antioxidante de extratos de bagaco de uva (Vitis vinifera) variedades Tannat e Ancelota. Ciência e Tecnologia de Alimentos, v. 28, n. 1, p. 238-244, 2008. DOI: https://doi.org/10.1590/S0101-20612008000500036

SILVA, A. B.; WIEST, J. M.; CARVALHO, H. H. C. Compostos químicos e atividade antioxidante analisados em Hibiscus rosa-sinensis L. (mimo-de-vênus) e Hibiscus syriacus L. (hibisco-da-síria). Brazilian Journal of Food Technology, v. 19, e2015074, 2016. DOI: https://doi.org/10.1590/1981-6723.7415

TEIXEIRA, L. N.; STRINGHETA, P. C.; DE OLIVEIRA, F. A.; Comparação de métodos para quantificação de antocianinas. Revistas Ceres, Viçosa - MG, v. 55, n. 4, p. 297304, 2008. DOI: 55(4): 297-304, 2008. 